\title{
DESCUBRIMIENTO DE INFORMACIÓN CIENTÍFICA: ¿TODAVÍA MISIÓN Y VISIÓN DE LA BIBLIOTECA ACADÉMICA?
}

\section{Scientific information discovery: Still a vision and a mission of the academic library?}

\author{
Blanca Rodríguez-Bravo, Maria-da-Graça Simões, Maria-Cristina Vieira-de- \\ Freitas y José-Antonio Frías
}

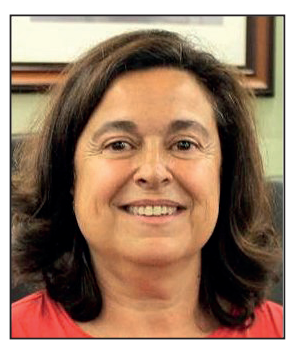

Blanca Rodríguez-Bravo es profesora titular de universidad en el Área de Biblioteconomía y Documentación en la Universidad de León. Sus intereses científicos se dirigen al estudio de la utilización de revistas electrónicas, el desarrollo de colecciones, la comunicación científica y la organización del conocimiento. Ha dirigido proyectos de investigación y ha publicado trabajos relacionados con estos campos en libros, revistas científicas y actas de congresos.

http://orcid.org/0000-0002-9476-7602

Universidad de León, Área de Biblioteconomía y Documentación Departamento de Patrimonio Artístico y Documenta Campus de Vegazana, s/n. 24071 León, España blanca.rodriguez@unileon.es

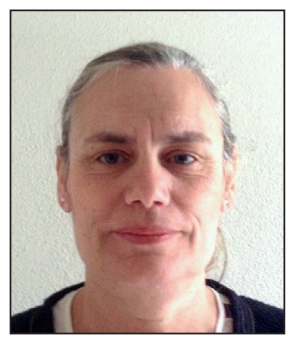

Maria-da-Graça Simões es profesora auxiliar en el Departamento de Filosofía, Comunicación e Información, Facultad de Letras, Universidad de Coimbra (Portugal). Sus intereses investigadores y docentes se centran en la organización del conocimiento. Ha publicado trabajos relacionados con este campo en libros, revistas científicas y actas de congresos.

http://orcid.org/0000-0001-5575-0261

Universidade de Coimbra, Faculdade de Letras Largo da Porta Férrea, Coimbra 3004-530, Portugal gsimoes@fl.uc.pt

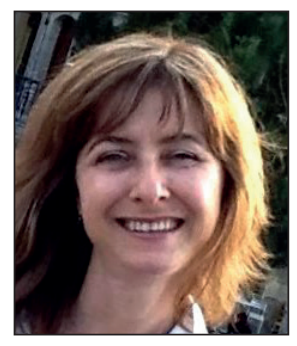

Maria-Cristina Vieira-de-Freitas es profesora auxiliar invitada en el Departamento de Filosofía, Comunicación e Información, Facultad de Letras, Universidad de Coimbra (Portugal). Sus intereses investigadores y docentes se centran en la organización del conocimiento en el campo de los archivos, la teoría y metodología archivística, la investigación cualitativa y el análisis cualitativo de datos asistido por ordenador. Ha publicado trabajos relacionados con estos campos en libros, revistas científicas y actas de congresos.

http://orcid.org/000-0002-8849-8792

Universidade de Coimbra, Faculdade de Letras Largo da Porta Férrea, Coimbra 3004-530, Portugal cristina.freitas@fl.uc.pt

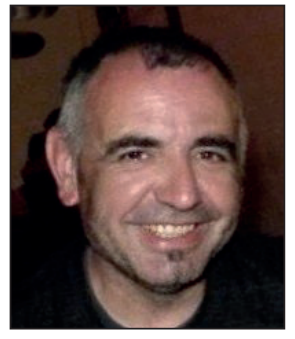

José-Antonio Frías es profesor titular de universidad, adscrito al Departamento de Biblioteconomía y Documentación de la Universidad de Salamanca. Sus intereses investigadores son la comunicación científica, las metodologías de investigación y los estudios de género en información y documentación. Ha dirigido tesis doctorales y proyectos de investigación y ha publicado trabajos relacionados con estos campos en libros, revistas científicas y actas de congresos.

http://orcid.org/0000-0002-5425-8950

Universidad de Salamanca, Departamento de Biblioteconomía y Documentación, Facultad de Traducción y Documentación Francisco de Vitoria, 6. 37008 Salamanca, España frias@usal.es

\section{Resumen}

El acceso a contenidos de calidad es clave para la investigación y uno de los valores principales que los académicos asignan a la biblioteca. Los datos bibliográficos desempeñan un papel trascendental en las bibliotecas universitarias, que dedican abundantes recursos a su obtención y disposición para el acceso. El estudio indaga sobre dónde y cómo se descubre la infor- 
mación bibliográfica, y pone de manifiesto el papel de los motores de búsqueda, bases de datos, repositorios y servicios de descubrimiento en ese proceso. El esfuerzo que las bibliotecas han realizado implementando estos servicios parece haber dado sus frutos en relación al incremento en el uso de las colecciones. Sin embargo, Google parece conservar la prioridad como opción de descubrimiento de información científica. Se trata de un estudio de revisión, basado en el análisis de investigaciones originales, así como de resultados difundidos en informes recientes.

\title{
Palabras-clave
}

Bibliotecas académicas; Bibliotecas universitarias; Búsqueda de información; Comportamiento en la búsqueda; Descubrimiento de información; Información científica; Servicios de descubrimiento.

\begin{abstract}
Access to quality content is key to research and one of the core values that scholars assign to the library. Bibliographic data play a fundamental role in university libraries, which devote abundant resources to obtaining and hosting them for access. This study investigates where and how bibliographic information is discovered, and highlights the role of search engines, databases, repositories, and web-scale discovery services in that process. The effort that libraries have made in implementing these services seems to have paid off in relation to the increase in the use of collections. However, Google remains the top option for discovering scientific information. This is a review study, based on the analysis of original research and results from recent reports.
\end{abstract}

\section{Keywords}

Academic libraries; Discoverability; Information seeking; Information seeking behaviour; Scientific information; University libraries; Web-scale discovery services.

Rodríguez-Bravo, Blanca; Simões, Maria-da-Graça; Vieira-de-Freitas, Maria-Cristina; Frías, José-Antonio (2017). “Descubrimiento de información científica: ¿todavía misión y visión de la biblioteca académica?". El profesional de la información, v. 26, n. 3, pp. 464-479.

https://doi.org/10.3145/epi.2017.may.13

\section{Introducción}

El acceso inmediato a contenidos de calidad es clave para la investigación y es el valor principal que los académicos asignan a la biblioteca, como se desprende de un informe de Research Information Network (RIN) (2011). Los datos bibliográficos han desempeñado un papel trascendental en las bibliotecas universitarias que necesitan cumplir su misión de apoyo a la investigación, la enseñanza y el aprendizaje. Con esta finalidad, las bibliotecas académicas dedican abundantes recursos a la adquisición, suscripción, gestión y creación de datos para facilitar a sus usuarios el acceso a los contenidos disponibles.

Una de las finalidades de las bibliotecas instalando servicios de descubrimiento ha sido recuperar el protagonismo perdido frente a Google

Sin embargo, Schonfeld (2014) cuestiona si es adecuado que las bibliotecas universitarias consideren su misión el ser punto de partida en el viaje de la búsqueda de información. Según este autor, las bibliotecas desean ser consideradas como un valor añadido en el paisaje rápidamente cambiante de la información y justificar así su posición dentro de la institución, en vez de conformarse con ser intermediarias de acceso a los contenidos suscritos por sus instituciones.

Como señala Ann Rossiter, directora ejecutiva de la Society of College, National and University Libraries (Sconul), las bi- bliotecas trabajan para conseguir proporcionar acceso fácil a los contenidos y, así, no es trascendente si el descubrimiento se realiza vía Google o por medio de los servicios de descubrimiento, ni si se accede a ellos de forma remota o visitando la biblioteca en persona (cit. por Else, 2016).

Asimismo, Ávila, Ortiz y Rodríguez-Mateos (2015) se preguntan si las bibliotecas deben amoldarse y replicar el modelo de búsqueda de información propio de la Web o deben centrarse en mejorar, dar valor a sus propios sistemas y esforzarse en la alfabetización informacional de los usuarios. En una palabra, la cuestión reside en si las bibliotecas tienen o no que competir con Google por la atención de los usuarios.

Es frecuente la argumentación de que la búsqueda de información científica no se resuelve a través de Google y que las bibliotecas y sus herramientas están más preparadas para facilitar ese tipo de información. Como señalan Spezi, Creaser y Conyers (2015) los bibliotecarios son más positivos acerca de las posibilidades de los servicios de descubrimiento que los editores y que los proveedores de contenido, que son escépticos sobre su impacto en el uso de la información.

Igualmente, parece que las generaciones de jóvenes investigadores muestran preferencia por los motores de búsqueda y las bases de datos más reputadas en detrimento de otras herramientas bibliotecarias, como se deriva de los resultados provisionales del proyecto Harbingers (Nicholas, 2016) ${ }^{1}$ que indaga sobre las actitudes de los investigadores noveles en el proceso de la comunicación académica.

En respuesta a estos resultados preliminares del proyecto citado, Martin Reid, director de la biblioteca de la London 
School of Economics, reconoce que los investigadores son más autónomos y que el papel de la biblioteca está cambiando en su relación con ellos, tratando de cubrir nuevas áreas en la cadena de la comunicación científica (cit. por Else, 2016). Así, las actuales bibliotecas académicas se vuelcan en el apoyo a la gestión de datos, a la disposición de sus contenidos en acceso abierto, a la gestión de los derechos de autor, en la orientación sobre el impacto y las métricas, etc.

El bibliotecario integrado deberá promover, además, el uso de los recursos electrónicos en la comunidad académica para establecer, como señala Anglada (2014), un nuevo estereotipo de biblioteca fundado en su papel de apoyo en el difícil proceso de usar la información y transformarla en conocimiento.

\section{La cuestión es si las bibliotecas tienen o no que competir con Google invirtiendo en servicios de descubrimiento}

En un trabajo anterior abordábamos las ventajas y desventajas de la búsqueda de información en opacs y servicios de descubrimiento (Rodríguez-Bravo et al., 2015). Ahora nos queremos preguntar por el acierto o desacierto de estos servicios de descubrimiento en la tentativa por reproducir la experiencia de búsqueda de la Web. Sin duda, la implementación de estos servicios refleja la preocupación de las bibliotecas por atender al usuario y conservarlo como tal.

Aunque el descubrimiento de conexiones entre ideas ha sido clave para el trabajo académico desde sus orígenes, el concepto de "discoverability" es relativamente nuevo (Conrad, 2017b). Hace referencia a la facilidad para ser encontrado un ítem de información. El descubrimiento se relaciona con la búsqueda de información y el acceso a dicha información pero no se trata de conceptos equivalentes. La búsqueda es previa al descubrimiento y el acceso a la información es posterior a su descubrimiento. Además, el "descubrimiento de" o "encuentro con" los contenidos puede producirse por rutas o canales informales y ajenos a la búsqueda. Así, apunta Akeroyd (2017) que el descubrimiento de información es un combinado de búsqueda deliberada y serendipia. Como Warren (2015) indica, los metadatos son clave para un descubrimiento efectivo de información.

En las bibliotecas el propósito de facilitar al usuario el encuentro con la información ha llevado a desarrollar un conjunto de sistemas bien asentados que proporcionan acceso a la colección. Es el caso de los catálogos, las listas A/Z, las guías de recursos, o los servicios de descubrimiento, entre otros. Sin embargo, el acceso y el descubrimiento han sobrepasado el nivel local de nuestras bibliotecas y han escalado a la Web. El reto no reside sólo en mejorar los sistemas locales sino en conseguir que los recursos bibliotecarios puedan ser descubiertos en otras sedes y sistemas. Se trata, como señalaba Dempsey (2013), de que estén disponibles donde los usuarios tienen sus experiencias de descubrimiento. Ello incluye motores de búsqueda, redes académicas o librerías como Amazon.
El presente estudio trata de determinar dónde y cómo encuentra la comunidad académica la información científica y pone de manifiesto el papel de las bibliotecas académicas en la búsqueda y localización de información por medio de la implementación de servicios de descubrimiento.

Se trata de una revisión bibliográfica, basada en el análisis de un conjunto de investigaciones originales de relieve internacional, así como de informes de proyectos recientes. De su estudio se extrae un conjunto de reflexiones útiles para la comprensión de las tendencias más actuales sobre los canales utilizados por los usuarios para encontrar la información científica que requieren.

El trabajo se estructura en dos grandes apartados. El primero aborda la diversidad de los canales de descubrimiento de información y las preferencias del usuario. El segundo se centra en la relevancia de los servicios de descubrimiento de la biblioteca como lugar de inicio de la búsqueda bibliográfica. A estos dos apartados se añade un tercero que contiene unas reflexiones finales.

\section{Dónde y cómo encuentra la comunidad académica la información científica}

El entorno de la información electrónica ha modificado drásticamente los comportamientos informativos en el sector de la educación universitaria. Durante los últimos 15 años se han llevado a cabo numerosos estudios sobre la evolución del comportamiento de los usuarios de las bibliotecas en la búsqueda de información, principalmente de los estudiantes, y de su adaptación al cambiante orden de la comunicación.

Como señalan Nicholas et al. (2017), encontrar, buscar o descubrir información deben ser entendidos en el contexto del nuevo orden informativo. En un entorno global y de información abierta, en el que la búsqueda puede llevarse a cabo en cualquier lugar y en cualquier momento, el comportamiento de los usuarios de información científica está cambiando. Buscar todo tipo de productos es tan habitual en el espacio virtual, que está dejando casi de ser una práctica consciente.

Para facilitar al usuario el encuentro con la información las bibliotecas han implementado catálogos, listas $A / Z$, guías de recursos, y servicios de descubrimiento, entre otros

Diversas investigaciones muestran que cada vez es más frecuente que los usuarios no comiencen el descubrimiento de información en el portal de la biblioteca sino en Google, Google Scholar o plataformas similares (OCLC, 2009; Inger; Gardner, 2013; 2016). Asimismo, los flujos de trabajo se han modificado. Se han trasladado desde recursos locales, como la biblioteca, a recursos en red, plataformas albergadas por internet (Dempsey, 2012). Esta realidad manifiesta que la biblioteca universitaria se ha convertido, para algunos usuarios, en el último recurso (ACRL Research Planning 
and Review Committee, 2012). Acuden a la biblioteca, física o virtual, sólo si no han conseguido el documento que necesitaban por otro medio (Spezi; Creaser; Conyers, 2015).

No obstante, los resultados de las 40.439 encuestas realizadas a lectores de publicaciones académicas de todo el mundo difundidos en el último informe de Gardner e Inger (2016), que reproduce las preguntas de estudios desarrollados desde 2005 con periodicidad trianual, ponen de relieve que el descubrimiento de contenido sigue constituyendo un espacio muy competitivo donde no existe un claro ganador. Asimismo, Conrad (2017a), tras analizar 13 documentos recientes sobre los canales utilizados para encontrar información concluye que las tendencias fluctúan según el tipo de usuario y sus necesidades de información, así como en función de las diversas variables demográficas.

Los usuarios acuden a la biblioteca, física o virtual, sólo si no han conseguido el documento que necesitaban por otro medio (Spezi et al., 2015)

Según Gardner e Inger (2016) la clave de las diferencias en el comportamiento de los lectores parece residir en el conocimiento de los recursos disponibles para facilitar el descubrimiento de la información. Este conocimiento es mayor en el sector académico y en las naciones más ricas donde se lleva a cabo una mayor labor de alfabetización informacional y existe un mayor abanico de opciones de descubrimiento.

Por su parte, Warwick et al. (2009) en su estudio longitudinal sobre los estudiantes universitarios han demostrado que los estudiantes tienen una marcada preferencia por el uso de internet como fuente de información con respecto a la utilización de los más complejos instrumentos bibliotecarios para acometer las tareas académicas. Es más, los estudiantes muestran su satisfacción reproduciendo estrategias de búsqueda que han resultado adecuadas anteriormente porque les han proporcionado suficiente información para sus necesidades en poco tiempo, y se muestran poco dispuestos a desarrollar sus habilidades en la búsqueda de información más allá de lo que consideran estrictamente necesario -siempre que no se vean exigidos por una tarea concreta-.

El estudio de Perruso (2016) se focaliza, así mismo, en los estudiantes universitarios, y muestra que estos prefieren la utilización de Google, si bien se constata que según van avanzando en su carrera tienden a utilizar más los recursos de la biblioteca. Para ello resultan determinantes tanto la formación impartida por los bibliotecarios como las exigencias de sus profesores.

Los informes Ithaka en los que se recogen los resultados de encuestas realizadas en el mundo anglosajón también son una fuente de datos para conocer la evolución en este campo. Así, el informe de 2013 (Long; Schonfeld, 2014), basado en las respuestas de 499 directores de biblioteca de universidades americanas muestra un cambio en la prioridad de las bibliotecas que se están focalizando en la alfabetización informacional de los estudiantes más que en el apoyo a la investigación del personal docente e investigador, a quien se considera más autónomo en este terreno. Los últimos informes Ithaka realizados a partir de las encuestas al profesorado de EUA y Reino Unido en 2015 (Wolff; Rod; Schonfeld, 2016a; 2016b) confirman que también el profesorado considera prioritario el papel de la biblioteca en el fortalecimiento de las habilidades informacionales y de investigación de los estudiantes.

Spezi (2016) ha llevado a cabo una revisión de la literatura más reciente sobre el comportamiento de los estudiantes de doctorado, investigadores en ciernes, con respecto a la búsqueda de información. Los estudios revisados muestran estabilidad en las preferencias de este sector. En todas las disciplinas se constata el elevado uso de Google, mientras que la utilización de Google Scholar difiere dependiendo de las áreas. Se observan, sin embargo, sutiles cambios en el uso de las redes sociales y una tendencia al incremento en la utilización de las plataformas de e-recursos de las bibliotecas.

El ya citado proyecto Harbingers (Nicholas, 2016) basado en entrevistas a 116 jóvenes investigadores de 3 continentes y 7 países -entre ellos España- en los campos de ciencias y ciencias sociales, trata de indagar sobre si los jóvenes investigadores aprovechan las numerosas oportunidades de descubrimiento actualmente a su disposición -como es la presencia ubicua de su smartphone- en un mundo de la información cada vez más abierto, social y de fronteras borrosas.

Según van avanzando en su carrera, los estudiantes universitarios tienden a utilizar más los recursos de la biblioteca (Perruso, 2016)

Los estudios centrados en estudiantes de doctorado o jóvenes investigadores son relevantes habida cuenta de que se trata de conocer las actitudes de las nuevas generaciones de investigadores, sin duda aquellos con mayores habilidades tecnológicas, pues han crecido con internet proporcionándoles información con facilidad e inmediatez. Los principales resultados obtenidos del primer año de la investigación del proyecto Harbingers sobre descubrimiento de información científica son los siguientes (Nicholas et al. 2017):

- Google y Google Scholar son muy populares en cualquiera de las disciplinas y países en los que se realiza el estudio. Incluida China donde la utilización de Google no está autorizada.

- Las bibliotecas se utilizan para obtener el texto completo de los documentos, pero no se mencionan. Parece que los investigadores no son conscientes de que tienen acceso a muchos recursos gracias a las suscripciones mantenidas por sus universidades.

- Las bases de datos Web of Science (WoS) y Scopus son populares, bien conocidas y utilizadas.

- Las redes académicas y los smartphones se utilizan también para buscar información. 
El estudio de Pontis et al. (2015) basado en entrevistas a 61 investigadores de categorías y antigüedad variable indica que la experiencia determina los canales utilizados en la búsqueda de información. Así, los jóvenes investigadores, poco expertos, mayoritariamente buscan información relevante en la Web. Son los investigadores senior los que más valoran la interacción social con sus pares para mantenerse al día.

Tenopir et al. (2015) subrayan que los académicos están crecientemente diversificando sus canales de descubrimiento de información y que esta tendencia se ha incrementado con la aparición de las múltiples redes sociales.

\section{Los investigadores senior son los que más valoran la interacción social con sus pares para mantenerse al día (Pontis, 2015)}

Con respecto a los smartphones, Gardner e Inger (2016) curiosamente hallan que los lectores de países menos desarrollados utilizan los móviles para acceder a las revistas más que los entrevistados en los países más desarrollados. No obstante, apuntan que el acceso por medio del teléfono móvil supone sólo un $10 \%$ del uso total. Abundaremos a continuación en la utilidad de los diversos canales mencionados.

\subsection{Motores de búsqueda versus bases de datos}

La primacía en la búsqueda de Google/Google Scholar, puesta de relieve en numerosos estudios, entre ellos los que hemos mencionado, se confirma en el proyecto Harbingers. Google es la referencia en la búsqueda de información científica y los demás proveedores de acceso son cada vez más dependientes de su algoritmo para situarse en buena posición en su página. Como señalan Nicholas et al. (2017), la preferencia de los usuarios por Google no se basa sólo en la facilidad de su búsqueda sencilla, obedece, asimismo, a la creciente disponibilidad de información académica a texto completo y de libre acceso que deja al alcance del usuario.

El informe de Gardner e Inger (2016) destaca como uno de sus principales hallazgos que Google Scholar se utiliza más que Google en el sector académico, pero menos que Google en todos los restantes sectores. Google Scholar desempeña un papel principal sobre todo en el sector de STM (science, technology \& medicine) y en los países con mayor nivel de vida.

Las bases de datos WoS, Scopus y PubMed son mencionadas en prácticamente todos los países. El estudio citado de Gardner e Inger (2016), pone de relieve que las bases de datos bibliográficas, entre las que destacan WoS y Scopus, siguen siendo la principal fuente para el descubrimiento de la información científica aunque su utilización parece haber disminuido con respecto a los datos ofrecidos por las encuestas de 2012. Sin embargo, en los países más desarrollados los motores de búsqueda son el medio preferido cuando se trata de encontrar artículos de revistas. Se constata también un claro efecto PubMed Central en el sector médico.
Los resultados de la última encuesta de la serie Ithaka realizada al profesorado estadounidense en 2015 (Wolff; Rod; Schonfeld, 2016a) confirma la tendencia identificada en 2012, un descenso en la preferencia por las bases de datos bibliográficas y un aumento en la utilización de motores de búsqueda generalista, Google en particular. Ambos canales de descubrimiento de información se igualan en las preferencias del profesorado.

Borrego y Anglada (2016) una vez analizadas las respuestas a un cuestionario enviado a los investigadores catalanes, que sigue el modelo de la encuesta Ithaka $S+R$ de los EUA, encuentran que en el proceso de búsqueda de información la mitad de los encuestados afirma confiar en las bases de datos seguidas por los motores de búsqueda. En un estudio anterior en el ámbito catalán (Ollé; Borrego, 2010) se mostraba la preferencia de los académicos de las universidades catalanas por encontrar la información científica a través de Google.

Otros estudios realizados en la Península Ibérica (RodríguezBravo et al., 2013; Rodríguez-Bravo; Alvite; Olea, 2015) basados en encuestas a docentes e investigadores con vinculación permanente y mayoritariamente de edad superior a los 40 años, de las universidades de Coimbra, León y Oporto, confirman la preferencia por buscar información a través de Google.

\section{La preferencia de los usuarios por Google no se basa sólo en su búsqueda sencilla sino también en que cada vez más faci- lita acceso a textos completos (Nicholas et al., 2017)}

La mayoría de los entrevistados en el estudio de Pontis et al. (2015) procedentes de Europa, América del Norte y Asia, prefieren también la búsqueda de información en la Web, principalmente utilizando Google. Secundariamente mencionan la utilización de PubMed, Google Scholar, las webs de las revistas y ResearchGate.

El análisis de Schonfeld (2015) ofrece similares resultados. Este autor afirma que Google y Google Scholar son puntos de acceso a la información muy relevantes; que los motores de búsqueda y los servicios de alerta de las principales plataformas de contenido desempeñan, asimismo, un papel reseñable. Indica también que las redes sociales académicas tienen una importancia creciente en el descubrimiento de la información. Sin embargo, los servicios de descubrimiento pueden desempeñar un papel en la búsqueda para determinados usuarios y determinadas prácticas pero no son primordiales para acceder a las grandes plataformas de contenidos.

El estudio de Faherty (2016) que se centra en el descubrimiento, evaluación y acceso a libros por parte de investigadores de ciencias humanas señala que los académicos de este sector también descubren información a través de Google, mientras que es menos frecuente la utilización de Google Scholar o Google Books. Los items ya conocidos, sin 
embargo, se localizan utilizando Amazon, las webs de los editores o los opacs.

Google Books sí es contemplado como un instrumento de búsqueda regular en todas las disciplinas analizadas en el estudio llevado a cabo con los doctorandos y los investigadores postdoctorales de la University of Cambridge (Priestner; Marshall, 2016).

\subsection{Repositorios institucionales y redes sociales}

Los repositorios institucionales no parecen jugar un papel protagonista en el descubrimiento de la información científica, pero sí conservan un interés para los académicos encuestados en el estudio mencionado de Gardner e Inger, fundamentalmente en los países menos desarrollados, donde las posibilidades de acceso a información de pago es limitada. Los resultados del último estudio Ithaka del Reino Unido (Wolff; Rod; Schonfeld, 2016b) reflejan un incremento en el depósito de la investigación en repositorios institucionales y temáticos que no se observa, sin embargo en las encuestas llevadas a cabo en Estados Unidos. La nueva actitud de los británicos puede guardar relación con la introducción de mandatos sobre el depósito de datos (Engineering and Physical Sciences Research Council).

https://www.epsrc.ac.uk

Los repositorios institucionales no parecen jugar un papel protagonista en el descubrimiento de la información científica

Los repositorios no parecen tampoco tener interés para los jóvenes investigadores entrevistados para el proyecto Harbingers. Los resultados muestran que la mayoría de ellos no tiene hábito de autoarchivar. Quienes tienen constancia de la existencia de un repositorio en su institución, suelen responder que son los bibliotecarios quienes se encargan de poner en acceso abierto sus documentos.

Sin embargo, los repositorios institucionales han constituido otra de las apuestas de las bibliotecas para tratar de ser imprescindibles en el descubrimiento de información. Su misión consiste en facilitar, en acceso abierto y a texto completo, la producción propia de la universidad, tanto artículos científicos como materiales docentes que tradicionalmente no eran recuperados por los opacs. El análisis de los datos del mismo proyecto Harbingers (Nicholas et al., 2017) refleja un mayor interés por los repositorios temáticos, entre los que destaca el repositorio pionero ArXiv.

Tay (2016a) apunta las debilidades de muchos repositorios o agregadores que no enlazan o no indizan o no permiten reconocer qué referencias aportan texto completo, algo que resulta inadecuado en los tiempos actuales donde el acceso al texto completo es generalizado. Por el contrario, Google Scholar permite identificar con claridad si existe o no acceso al texto completo además de indizarlo y facilitar el descubrimiento de información en su contexto. Tay indica, asimismo, que los repositorios carecen de una alarmante falta de normalización de datos, y que esa laxitud -uso del mínimo de etiquetas de Dublin Core- les está perjudicando porque la recuperación resulta en un conjunto inconsistente de items.
Es decir, que tampoco en este aspecto compiten en superioridad de condiciones con los motores de búsqueda y están a años luz de los pertinentes resultados que se obtienen en las tradicionales bases de datos que hemos citado.

La popularidad de las redes sociales digitales científicas es, por el contrario, un hecho. Pensamos que las posibilidades que ofrecen las aplicaciones sociales académicas como medios de comunicar, compartir y como escaparates donde exhibir la investigación puedan ser responsables, al menos en parte, del éxito limitado del uso de los repositorios institucionales. Tay (2016a) pone de relieve la fuerte competencia que los repositorios afrontan con las redes sociales académicas como ResearchGate.

Los jóvenes investigadores entrevistados en el proyecto Harbingers (Nicholas et al., 2017) mencionan la utilización de Facebook, Twitter, YouTube y LinkedIn, entre otras redes sociales. Con respecto a las redes académicas, las citadas principalmente son Academia.edu y ResearchGate, esta última de uso generalizado entre investigadores de ciencias y ciencias sociales. Así lo confirman también Orduña, Martín y Delgado (2016) apuntando que cuenta con más de 9 millones de usuarios y 80 millones de documentos.

El estudio de Nicholas, Herman y Jamali (2015) presentaba los resultados de un proyecto ${ }^{2}$ basado en las entrevistas a un centenar de académicos de varios países europeos y cuatro disciplinas en relación a la reputación académica en la era de la ciencia 2.0. En él se prestaba especial atención al papel de las redes académicas en la construcción y mantenimiento de la reputación de los investigadores. De este proyecto se derivan dos ideas principales:

- que las plataformas académicas se utilizan principalmente para compartir resultados de la investigación, sobre todo artículos y comunicaciones de congresos; y

- que la mayoría de los usuarios no saca provecho de los aspectos sociales de las redes.

Los repositorios carecen de una alarmante falta de normalización de datos, y su uso mínimo de etiquetas DC da lugar a búsquedas poco precisas (Tay, 2016a)

Es decir, interesan las posibilidades que ofrecen las redes para acceder a documentos de los colegas, e interesa también la visibilidad que pueden proporcionar y su repercusión en descargas, lecturas y quizá citas. Por el contrario, no interesa demasiado la interactuación con pares, ni la colaboración con otros investigadores a través de estas redes. Seguramente se trata de una cuestión de tiempo y los académicos terminarán aprovechando todas las funciones, y siendo más activos en las redes por dos razones principales:

- porque diversos estudios parecen indicar la repercusión en el número de citas de los trabajos difundidos a través de estas redes, véase el trabajo sobre la red Academia. edu de Niyazov et al. (2016);

- porque la interactividad en estas redes tiene una elevada repercusión en las métricas obtenidas, como ponen de re- 
lieve Orduña, Martín y Delgado (2016) y Nicholas, Clark y Herman (2016) en relación a ResearchGate.

Gardner e Inger (2016) hallan que es en los países más desfavorecidos donde cobra mayor relieve el descubrimiento de información y la obtención de documentos a través de las redes sociales que, en general, han ido incrementando su papel. Asimismo, Wolff, Rod y Schonfeld (2016b) indican que existe una tendencia entre los profesores británicos a incrementar el uso de las redes sociales para actualizarse en su disciplina en detrimento de la consulta regular o la recepción de alertas de los sumarios de las revistas.

\subsection{Bibliotecas universitarias y editores}

El informe de Gardner e Inger (2016) pone de relieve las diferencias en el descubrimiento de información entre las disciplinas, siendo los investigadores de humanidades y ciencias sociales los más afines a la utilización de la biblioteca como punto de partida para el descubrimiento de la información científica. Se aprecia, sin embargo, un cierto declive en la utilización de la biblioteca como fuente principal entre los resultados de 2012 y 2015.

Afirman estos autores que de las encuestas llevadas a cabo en 2015 se desprende que los investigadores de los países más desarrollados valoran tan positivamente las bibliotecas como portales de descubrimiento como las bases de datos bibliográficas, y se observa un papel significativo en concreto de los servicios de descubrimiento, aunque no han cobrado más relieve del que tenían en 2012.

Por su parte, el último informe Ithaka realizado en los EUA (Wolff; Rod; Schonfeld, 2016a) indica que la web de la biblioteca y el catálogo han cobrado mayor importancia para los usuarios que la que se reflejaba en el cuestionario de 2012. De hecho la percepción del papel de la biblioteca en la búsqueda de información alcanza los niveles que tenía en 2003. Los autores se preguntan si esta nueva visión más positiva guardará relación con la implementación de los servicios de descubrimiento en las bibliotecas universitarias.

Esta percepción relativamente positiva del papel de la biblioteca no es confirmada por los resultados del proyecto Harbingers. De la lectura de los datos recabados se obtiene la impresión de que las bibliotecas están perdiendo visibilidad por lo que a los jóvenes investigadores se refiere (Nicholas et al., 2017). Sus catálogos y sus esperanzadores servicios de descubrimiento no son una prioridad para los investigadores noveles. Así, aunque los investigadores a menudo consiguen el acceso a las bases de datos y a los proveedores de contenidos electrónicos a través de su biblioteca no se menciona la web de la biblioteca como un medio de descubrimiento de la información. Las bibliotecas, si acaso, son vistas como facilitadoras de acceso pero no de descubrimiento.

Esta visión poco halagüeña de la biblioteca se muestra parcialmente en otros estudios que no se focalizan en una franja de edad sino que abarcan a todo el personal docente e investigador. Así, en el ámbito catalán, Borrego y Anglada (2016) obtienen como resultado que el porcentaje de académicos que utiliza el catálogo de la biblioteca es inferior al que utiliza las bases de datos o los motores de búsqueda, y que la opción de visitar la biblioteca es elegida muy ocasionalmente. Sin embargo, para la localización de documentos de los que ya se tiene la referencia, el catálogo de la biblioteca o su web son las fuentes principales.

Este papel del opac en el acceso a documentos conocidos se refleja en el estudio de Faherty (2016). Sin embargo, este trabajo recoge la frustración de los académicos de humanidades con el catálogo como mecanismo de descubrimiento y se pregunta si las bibliotecas no tendrían que dejar de perseguir representar un papel en el descubrimiento de información científica.

Faherti, por el contrario, confirma el papel en el descubrimiento de la información de los editores. Respecto a los principales proveedores de información electrónica, Gardner e Inger (2016) recogen que ScienceDirect es el más utilizado, algo que no sorprende pues ha sido comprobado a través de las métricas de uso en todos los ámbitos geográficos incluidos el español (Rodríguez-Bravo; Alvite, 2011).

\section{Los investigadores de humanidades y ciencias sociales son los más afines a la utilización de la biblioteca para el des- cubrimiento de la información científica (Gardner; Inger, 2016)}

El estudio de Nicholas et al. (2017) muestra que el acceso a la información a través de los proveedores de contenidos electrónicos no es común en ninguna de las disciplinas abarcadas en el proyecto Harbingers, que incluyen la mayoría de ellas, exceptuadas las humanidades. Sin embargo, los jóvenes investigadores sí utilizan alertas en las bases de datos y distribuidores de publicaciones electrónicas y siguen citas y referencias para su actualización sobre la información publicada. También el estudio de Ollé y Borrego (2010) corrobora el uso de las alertas entre los académicos de las universidades catalanas.

Igualmente, Gardner e Inger (2016) destacan el rol de las alertas como medios de descubrir información y confirman el papel que desempeñan también las webs de los editores como recursos de búsqueda cada vez más populares. Atribuyen el avance en la utilización de las webs de las grandes empresas distribuidoras de contenidos en el proceso de búsqueda de información a la mejora de sus interfaces. En este particular ese estudio contradice los resultados del proyecto Harbingers donde prácticamente sólo se concede relieve a Elsevier (Nicholas et al., 2017).

El distanciamiento con la biblioteca física queda confirmado en los diversos estudios consultados. Por ejemplo el de Pinto, Fernández-Marcial y Gómez-Camarero (2010), que recoge datos de un cuestionario enviado a 19 universidades españolas y concluye que el personal académico prefiere acceder a los documentos online y sólo visita ocasionalmente la biblioteca. Ya en 2008 Nicholas alertaba sobre el éxodo de los usuarios desde las bibliotecas hacia los editores, que se aceleraría con el desarrollo de la oferta bibliotecaria de e-books al sumarse al proceso migratorio los estudiantes y 
los investigadores de las áreas de ciencias sociales y humanidades, menos proclives al uso de las revistas.

Ante este desapego que muestran los investigadores respecto a la biblioteca queremos preguntarnos si la implementación de los web-scale discovery services ha servido, en alguna medida, para fidelizar a los usuarios en las bibliotecas por lo que se refiere a su rol de mediación en el descubrimiento de la información.

\section{Utilidad e impacto de los servicios de descubrimiento para la localización de nuevos recursos}

Como apunta Rodríguez-Yunta (2015), los argumentos que apoyan la adopción de los servicios de descubrimiento son dos:

- la insatisfacción de los usuarios con las prestaciones del opac tradicional superado por los buscadores y otros recursos accesibles online, hasta el punto de dejar de ser útil incluso para acceder al fondo accesible a través de la biblioteca;

- los usuarios demandan interfaces de consulta con un diseño similar a las de los buscadores de internet. Dado que el usuario rechaza la consulta de diferentes recursos utilizando diversas interfaces se presupone que estará satisfecho si el catálogo le facilita la consulta de un abundante número de fuentes con una única estrategia de búsqueda.

Asimismo, el lector demanda que los catálogos actúen como bibliotecas digitales dando acceso al texto completo de los documentos, sin intermediación, puesto que, como se ha señalado, la biblioteca pretende conservar, al menos, su papel como proveedora de contenidos y con esa finalidad suscribe los paquetes de e-journals y e-books que los grandes editores ofertan a precios poco asequibles. El acceso abierto a documentos a texto completo está fortaleciendo el uso de Google y la biblioteca quiere mantener su posición en este terreno.

Las bibliotecas debieran continuar manteniendo las bases de datos temáticas y dejar las interfaces de descubrimiento para los motores de búsqueda gratuitos (Breitbach, 2016)

Se trata, por tanto, de conseguir que la biblioteca recupere protagonismo y ésta es la finalidad de los servicios de descubrimiento que se han implementado en gran número de bibliotecas universitarias. Son, pues, productos cuyo fin es conseguir que la biblioteca se constituya en la primera y principal fuente de información para el usuario, ser el lugar por el que éste inicie la búsqueda bibliográfica, como subraya Rodríguez-Yunta (2015).

Sin embargo, Breitbach (2016) desaconseja a las bibliotecas la inversión de sus limitados recursos en estos servicios de descubrimiento. En su opinión, las bibliotecas debieran continuar manteniendo las bases de datos temáticas y dejar las interfaces de descubrimiento para los motores de búsqueda gratuitos que son, en cualquier caso, utilizados ya mayoritariamente. En este escenario, los bibliotecarios continuarían poniendo el énfasis en la organización por materias de los fondos pero al mismo tiempo deberían priorizar que sus usuarios pudieran acceder con facilidad a los recursos descubiertos a través de Google Scholar vía Open URL, o métodos similares.

Con el fin de profundizar en la discusión, nos parece oportuno reflexionar sobre las características, las potencialidades y la utilidad de estos servicios en la búsqueda y localización de información agregada de distintas fuentes, por parte de los usuarios de las bibliotecas.

\subsection{Características definitorias de los servicios de descubrimiento}

Desde un punto de vista arquitectónico y visual, los servicios de descubrimiento, solución arbitrada por las bibliotecas para facilitar la búsqueda de todos los contenidos mediante una ventanilla única (Ávila; Ortiz; Rodríguez, 2015), son reconocibles principalmente por su utilización de la búsqueda facetada de un ítem catalogado que permite al usuario añadir o eliminar puntos de acceso y expandir o limitar su búsqueda. No obstante, más allá de las facetas, la utilidad de estos programas radica en la facilidad para combinar múltiples fuentes de metadatos en una única caja de búsqueda sencilla. La combinación de registros MARC, repositorios $O A l$, bases de datos, imágenes, materiales multimedia y otros materiales online conduce a la obtención exhaustiva de resultados hasta ahora nunca alcanzados de una manera tan sencilla (Rodríguez-Bravo et al., 2015).

El acceso desde la biblioteca a volúmenes de información ocultos anteriormente en silos de catálogos o bases de datos particulares, sin posibilidad de ser recuperados simultáneamente y desde un único punto de acceso, es una revolución tanto para los investigadores como para los usuarios inexpertos. Así, Breeding (2011) señala que los servicios de descubrimiento están pensados para encontrar, no sólo para buscar.

Como apunta Rodríguez-Yunta (2015), estos softwares han ido evolucionando desde mediados de los 90 a partir de los multibuscadores que ejecutaban búsquedas federadas. Los metabuscadores buscan en múltiples bases de datos y, posteriormente, agregan los resultados. Con la utilización de software de búsqueda federada los resultados ofrecidos dependen tanto de los algoritmos de búsqueda y de los rankings de relevancia del metabuscador como de los correspondientes a cada herramienta utilizada.

Contrariamente, los servicios de descubrimiento importan los metadatos a un único índice y aplican un único conjunto de algoritmos de búsqueda y una única fórmula de ordenación de resultados. Los catálogos se extienden para incluir búsquedas a artículos de revistas (WorldCat) y grandes índices de artículos de revistas engloban catálogos de bibliotecas y repositorios institucionales (Ebsco Discovery Service (EDS), Primo Central, Summon). Cualquiera de las dos rutas conduce al resultado deseado de ofrecer un acceso unificado a todos los recursos que la biblioteca posee o contrata (Anglada, 2012). 
Es a partir de 2009 cuando surge una nueva generación de servicios de descubrimiento que se suelen denominar web-scale discovery services o index-based discovery services. Como señala Breeding (2015b) se han convertido en componentes vitales de la infraestructura de la biblioteca académica y siguen cosechando ventas, tanto primeras implementaciones como cambios de proveedor. Señala este autor que de este modo las bibliotecas universitarias tratan de optimizar el impacto de sus inversiones en recursos electrónicos, consiguiendo generar un mayor consumo, necesidad puesta de relieve en numerosos estudios que se han basado en las estadísticas de descargas de los principales proveedores de recursos electrónicos, sirvan de ejemplo los trabajos de Ciber ${ }^{3}$ en el ámbito anglosajón.

El camino no está, sin embargo, exento de obstáculos. El índice centralizado de los servicios de descubrimiento precisa de la existencia previa de acuerdos con los proveedores comerciales de los productos bibliográficos, a fin de poder integrar los recursos suscritos por las bibliotecas. En este punto surgen conflictos entre las empresas que distribuyen contenidos y software de descubrimiento (Kennedy, 2014), y existe una evidente falta de transparencia en la cobertura de fuentes que ofrece cada uno de los programas como ponen de relieve Ávila, Ortiz y Rodríguez-Mateos (2015), entre otros autores.

Asimismo, se producen vacíos en la distribución de contenidos como evidencia el estudio de Zhu y Kelley (2015) realizado desde el punto de vista de un editor, IEEE Xplore. En él se pone de manifiesto que no todos los proveedores proporcionan contenidos completos y actualizados a los servicios de descubrimiento; que los contenidos proporcionados no siempre son indexados en su totalidad por los servicios; y que algunas bibliotecas implementan sus programas incorrectamente, impidiendo la exposición del contenido completo del editor.

Así, señala Schonfeld (2015) que si los servicios de descubrimiento quieren convertirse en el principal punto de entrada a las colecciones tienen que actualizar los contenidos con mayor frecuencia. No tiene sentido que Google Scholar ofrezca contenidos recién publicados antes que el proveedor del índice del servicio de descubrimiento.

\subsection{Impacto de los servicios de descubrimiento}

La implantación de los servicios de descubrimiento ha sido generalizada en los últimos años, pero su éxito está todavía por confirmar. El efecto más deseado por los bibliotecarios al adoptar uno de tales servicios es, como es lógico, que aumente el acceso a las colecciones, como apuntaba Breeding (2015b). Los bibliotecarios de universidades españolas sostienen que se ha conseguido ese objetivo en las respuestas a la encuesta distribuida por Ávila-García (2013). En ella el $66 \%$ de los centros confiesa no disponer de datos pero un $30 \%$ responde que el uso de la colección ha aumentado. Diversos estudios parecen ratificar esta afirmación, como procedemos a exponer a continuación.

En el ámbito americano se han llevado a cabo numerosos estudios de caso. Así, Way (2010) analizó los cambios en las estadísticas de uso de la colección durante los 6 meses posteriores a la adopción de Summon por parte de las bibliotecas de la Grand Valley State University con el fin de determinar su impacto. Los resultados muestran un acusado descenso en el uso de bases de datos especializadas que ofrecen servicios de resúmenes e indización y un espectacular aumento del uso de las bases de datos a texto completo, las revistas electrónicas y el acceso a recursos mediante un servidor de enlaces. En opinión de este autor, estos datos sugieren un impacto muy positivo de Summon en el uso de la colección.

También O'Hara (2012) confirma el incremento de las descargas tras la puesta en funcionamiento de Summon en la University of Manitoba. Chapman (2012) asevera que los servicios de descubrimiento pueden desempeñar un papel significativo en la atracción de los usuarios hacia la biblioteca. Su trabajo muestra que en la University of Michigan los usuarios están igual de satisfechos con Summon que con Google Scholar.

Kemp (2012), por su parte, realizó un estudio similar de las estadísticas de uso de la colección de las bibliotecas de la University of Texas tras instalar Summon en 2010. Los resultados son muy similares a los de los estudios citados. Durante el primer año de uso de Summon, el acceso a los recursos electrónicos aumentó notablemente: las descargas de artículos a texto completo se incrementaron un $23 \%$ y el uso de servidores de enlaces subió un $84 \%$. Por el contrario, el uso del opac disminuyó un $14 \%$ y el acceso a bases de datos bibliográficas cayó un 5\%. De nuevo, los resultados apuntan al efecto favorable de la adopción de un servicio de descubrimiento. Asimismo, Faiks, Oberg y Gabrio (2012) constatan la existencia de un impacto positivo en las estadísticas de circulación de documentos y en el préstamo interbibliotecario tras la implementación de WorldCat Local en el Macalester College, Saint Paul, Minnesota.

En el entorno británico, Thoburn, Coates y Stone (2012), que estudian el impacto del servicio de descubrimiento de Summon en las Universidades de Huddersfield y Northumbria, refieren que la implementación de un servicio de descubrimiento tiene repercusión en un incremento en el uso de las revistas electrónicas y en una disminución de la utilización de las bases de datos de resúmenes e índices.

Lam y Sum (2013) informan de una influencia positiva en el uso de los recursos después de la implementación de Primo en la Open University of Hong Kong. Otros estudios de caso, algunos comparativos entre programas con resultados similares que podemos citar son los llevados a cabo por Baldwin et al. (2012); Kaufmann, Larsen y De-Salvo (2012); Asher, Duke y Wilson (2013); Mussell y Croft (2013) y Djenno et al. (2014). Para profundizar en la materia es recomendable acudir a la exhaustiva bibliografía actualizada elaborada por François Renaville: Discovery tools: A bibliography ${ }^{4}$.

No queremos dejar de mencionar algunos estudios de mayor cobertura como es el de Levine-Clarl, Price y McDonalds (2013) que extiende el análisis a un grupo de instituciones y servicios de descubrimiento tratando de indagar en el impacto que su implementación tiene en el uso de revistas. Para ello seleccionaron 20 bibliotecas, americanas en su mayoría, que disponen de alguno de los cuatro principales 
servicios de descubrimiento: EDS, Primo Central, Summon y WorlCat Local. El estudio revela que ningún servicio de descubrimiento tiene un efecto claro en el aumento o la disminución del uso de los recursos en todas las bibliotecas ni con respecto a todos los editores. No obstante, sí se aprecian diferencias entre las herramientas.

El trabajo de Spezi, Creaser y Conyers (2015), que analiza el efecto de los servicios de descubrimiento en el uso de e-journals y e-books en bibliotecas de Reino Unido, muestra también que parece existir un efecto positivo en la mayoría de las instituciones estudiadas si bien las dimensiones de ese impacto varían según las bibliotecas y en función de los editores. Los datos obtenidos muestran un superior incremento en el uso de libros electrónicos que en el de revistas. Por su parte, el trabajo de Aznar (2014) presenta como resultados, basados en la revisión de diversos estudios los siguientes:

- Fuerte incremento en el uso de los recursos electrónicos a texto completo (tanto revistas como libros electrónicos), acompañado de una disminución en el uso del catálogo tradicional y el localizador de revistas electrónicas.

- Disminución en el uso de las bases de datos de artículos en su interfaz original. Es mucho mayor el número de registros de una determinada base de datos visualizados desde el servicio de descubrimiento que desde la base de datos original.

- Cambios en las políticas de desarrollo de la colección. Cuando una biblioteca evalúa una nueva suscripción a un recurso tiene en cuenta si está incluido en el índice del programa, pudiendo ser éste un factor determinante en la decisión de suscribir o no.

- Fuerte ascenso del préstamo interbibliotecario: en algunas bibliotecas hay un incremento de peticiones de materiales de otras bibliotecas de un $92 \%$ respecto a años anteriores.

- Competencia moderada con Google: aunque el buscador sigue siendo la primera opción (tanto en su versión académica como en la global), varios estudios indican que los usuarios consideran el servicio de descubrimiento como un complemento esencial en su proceso de búsqueda de información.

Breeding (2014) recoge la inquietud de los proveedores de servicios de resúmenes e indización documental, como Thomson Reuters/ISI -ahora Clarivate Analytics-, PubMed o Psychlnfo, sobre el descenso en el uso de sus bases de datos para enumerar las consecuencias de la instalación de un servicio de descubrimiento. Los tesauros especializados, resúmenes y demás productos documentales que aportan estas bases de datos son de gran valor para el proceso de búsqueda, pero la popularización de los servicios de descubrimiento podría afectar al interés que despiertan estos productos.

Los resultados preliminares del proyecto Harbingers, recogidos en Nicholas et al. (2017), sin embargo, contradicen esta percepción al mostrar el uso de WoS y de PubMed como recursos más habituales para el descubrimiento de información que los servicios de descubrimiento, no mencionados por ninguno de los 116 jóvenes investigadores entrevistados.
Ya hemos puesto de relieve más arriba que los estudios realizados a partir de cuestionarios (Gardner; Inger, 2016; Wolff; Rod; Schonfeld, 2016a) muestran un cierto optimismo acerca de la utilización de estas plataformas.

\subsection{Fortalezas y debilidades de los servicios de des- cubrimiento}

Rodríguez-Yunta (2015) pone de relieve las fortalezas y las debilidades de los servicios de descubrimiento. Como puntos fuertes señala:

- hacen efectiva la necesaria integración entre el catálogo y los recursos contratados disponibles;

- aportan mejoras en las prestaciones de búsqueda; y

- reproducen el formulario de búsqueda de Google que es el preferido por los usuarios.

Como puntos débiles indica que el servicio de descubrimiento es una nueva plataforma que no sustituye al catálogo. Se trata, por tanto, de una segunda opción que puede incrementar la desorientación del usuario que desconoce la diferencia opac/servicio de descubrimiento. Esta circunstancia fue indicada, asimismo, en el estudio publicado en 2014 por Rodríguez-Bravo et al.

Los servicios de descubrimiento se alejan de los catálogos y las bases de datos estructuradas de las bibliotecas en persecución de una quimera: competir con Google

Uno de los aspectos más relevantes del informe encargado por NISO a Breeding (2015a) es la revisión de los puntos débiles del mercado de los servicios de descubrimiento. Según Breeding, los siguientes aspectos todavía presentan carencias que sería preciso subsanar:

- Cobertura de los recursos: los índices centrales continúan poblándose con el objetivo de representar la totalidad de las colecciones bibliotecarias, pero todavía existen huecos.

- Internacionalización y cobertura de contenidos multilingües: la cobertura de recursos bibliográficos de fuentes de todo el mundo y en distintos idiomas está aún en proceso de crecimiento.

- Cobertura de materiales de acceso abierto: los principales servicios de descubrimiento están mejorando la cobertura de estos materiales, pero todavía es preciso avanzar en ello. Es de esperar que el interés de los desarrolladores vaya creciendo conforme aumenta el número de artículos de investigación publicados en fuentes de acceso abierto.

- Búsquedas de recursos ya conocidos y pertinencia de los resultados.

- Índices de relevancia: este factor es esencial, teniendo en cuenta la enorme cantidad de resultados que recuperan estas herramientas, y dado que los usuarios sólo exploran habitualmente los primeros resultados de la lista, obviando recursos relevantes. Se han hecho continuos progresos en este aspecto, pero no son suficientes. 
- Descubrimiento enriquecido mediante asociaciones no textuales: un ejemplo sería $b X^{5}$, que reutiliza la información de los patrones de uso de los servidores de enlaces para identificar recursos relacionados aunque no estén descritos con palabras clave similares. Esta técnica se basa en la probabilidad de que los artículos que un mismo investigador lee en una misma sesión estarán sujetos a algún tipo de relación. Este mecanismo es especialmente relevante si se introduce la disciplina investigada en el algoritmo de recomendación, ya que los patrones de uso de un investigador pueden servir como base para recomendar información de interés a otro usuario que investiga en el mismo ámbito científico.

- Interacción con los sistemas de gestión del conocimiento: la mayoría de los servicios de descubrimiento se ofrecen a través de la interfaz bibliotecaria, pero es esencial que se pueda acceder a su contenido y a sus funciones desde las interfaces de otros servicios familiares para los usuarios.

Por su parte, Breitbach (2016) apunta como debilidad la falta de consistencia en los metadatos y la ausencia de contexto en la relación de éstos con las disciplinas. Señala que los servicios de descubrimiento agregan datos con el fin de normalizarlos en un único índice y que ello trae como consecuencia que el valor de los metadatos disminuya. Estos sistemas no pueden competir con las bases de datos disciplinares en pertinencia de los resultados. La misma situación se ha puesto de relieve para los repositorios institucionales.

\section{El éxito de los servicios de descubrimien-} to en la misión de competir con Google parece cuestionable, pese a los estudios positivos de su impacto en el uso de la colección

Esta debilidad se ha tratado de mitigar con la creación de interfaces que facilitan la navegación facetada. Tales facetas cubren, sin embargo, tanto materias amplias como conceptos concretos, con lo que su utilidad es cuestionable (Breitbach, 2016; Rodríguez-Bravo et al., 2015). Para compensar la falta de una infraestructura robusta de metadatos, los servicios de descubrimiento han creado algoritmos que favorecen la exhaustividad en detrimento de la precisión.

En este sentido, señalan Freund, Poehlmann y Seale (2012) que el descubrimiento de los contenidos accesibles desde la biblioteca en cualquier formato debiera poder lograrse sin menoscabo de la pertinencia de la respuesta. Sin embargo, los resultados de trabajos empíricos cuestionan esta realidad (Fagan et al., 2012; Hofmann; Yang, 2012; Soules et al., 2014; Rodríguez-Bravo et al., 2015). Los servicios de descubrimiento se alejan, así, de los catálogos y las bases de datos estructuradas de las bibliotecas en persecución de una quimera: competir con Google.

Las posibilidades de interacción social no son una prioridad para las empresas proveedoras de estos servicios, y diversos estudios han comprobado sus limitadas funciones sociales (Rodríguez-Bravo et al., 2015; Pintos, 2016). Pintos (2016) ha observado el escaso uso del etiquetado social y las reseñas en los sistemas de descubrimiento implementados en las bibliotecas universitarias españolas. Sin embargo, la participación es frecuente entre los usuarios en otros entornos porque se percibe su utilidad para descubrir recursos. Este es el caso de las redes sociales académicas o de innumerables plataformas de servicios. Pintos se pregunta por qué no sucede así en las bibliotecas.

Breeding (2015a) opina que se debieran desarrollar opciones que concedan más oportunidades de interactuar socialmente en el propio programa de descubrimiento y pone como ejemplo el valor de la opinión experta de los investigadores en temas especializados para añadir puntos de acceso o indicar relaciones entre recursos que escapan a la catalogación/indización tradicional.

Apunta Race (2012) que los servicios de descubrimiento pueden facilitar el hallazgo accidental de recursos potenciando los aspectos sociales. También la navegación facetada puede facilitar la serendipia proporcionando medios que ayuden a visualizar las relaciones entre los resultados y potenciando aspectos subjetivos para determinar la relevancia, componente clave de la serendipidad.

\section{Reflexiones finales}

Encontrar información es, como se ha puesto de relieve, esencial para que los usuarios académicos desempeñen sus funciones, pero ese descubrimiento se está llevando a cabo crecientemente desde fuera de la biblioteca como subrayaban ya Tennant y RIN en 2009.

Se infiere que la preferencia de los académicos por Google/ Google Scholar como medio para la localización de información resulta incontestable. De acuerdo con Breitbach (2016), las bibliotecas se encuentran actualmente en desventaja en la confrontación con Google por el descubrimiento/búsqueda de la información. Es más, la preferencia por los motores de búsqueda generalistas trae aparejada una cierta invisibilidad de la biblioteca debido a que el usuario no es consciente de que si accede a gran número de recursos lo hace gracias a que sus bibliotecas universitarias los han suscrito.

No obstante, las bases de datos bibliográficas tradicionales suscritas por las universidades no han perdido protagonismo en el mundo de la información científica. Tanto el informe de Gardner e Inger (2016) como los resultados preliminares del proyecto Harbingers publicados en Nicholas et al. (2017) muestran la preferencia de los usuarios por las bases de datos WoS, Scopus y PubMed.

Asimismo, se observa un uso creciente de las redes sociales académicas que ayudan a construir la reputación de los investigadores como es el caso de ResearchGate, plataforma preferida en ciencias y ciencias sociales. Por el contrario, se constata entre las nuevas generaciones de investigadores un cierto desconocimiento y/o desapego sobre la utilidad de los repositorios institucionales, y ello pese a la existencia de sistemas recolectores de los contenidos archivados. Como apunta Tay (2016a) la agregación de repositorios no supone un avance imprescindible habida cuenta que los items de los repositorios son accesibles a través de Google y Google Scholar. 
Con respecto a los servicios de descubrimiento, su éxito en la misión de competir con Google parece cuestionable, pese a los estudios positivos de su impacto en el uso de la colección. En este contexto, Breitbach (2016) apunta que los web-scale discovery services están lejos de ser las eficientes plataformas de descubrimiento de contenidos soñadas. No obstante, cabe señalar que alguna de las herramientas presenta avances significativos. Tras una evaluación exhaustiva de 16 indicadores aplicada a los diferentes servicios de descubrimiento existentes en el mercado, Chickering y Yang (2014) indican que Primo tiene dos funcionalidades destacadas:

- la relevancia enriquecida por medio de la utilización de las estadísticas de uso; y

- la visualización de las relaciones FRBR (Functional requirements for bibliographic records).

No obstante, como apuntan Zhu y Kelley (2015) los vacíos de contenido en los programas de descubrimiento les restan credibilidad y reducir o cerrar esos huecos es una tarea continua que requiere la colaboración entre editores, proveedores de programas y bibliotecas. La checklist de NISO ODI (Open discovery initiative) publicada en 2015 proporciona un punto de partida para que las tres partes implicadas puedan cumplir ese objetivo. Subraya Schonfeld (2015) que es hora de asumir un mayor compromiso en el ecosistema de la información académica por parte de bibliotecas, editores e intermediarios en beneficio del usuario.

Como señalan Spezi, Creaser y Conyers (2015) los servicios de descubrimiento representan una inversión significativa para las instituciones, por tanto no necesitan sólo ser percibidos como una herramienta de valor añadido de la biblioteca con respecto a su institución, sino que deben demostrar ese valor con evidencias. Dado que su contratación es casi omnipresente existe una necesidad apremiante de entender el papel que representan tanto en el comportamiento ante la búsqueda de información, como en la gestión de los recursos bibliotecarios, como en su impacto en la utilización de contenido académico.

Como un área de mejora de estos servicios se apunta a la interacción social, aspecto destacado por Breeding (2015a) en su informe para NISO. Convenimos con Rodríguez-Yunta (2015) que para el mejor aprovechamiento de estas herramientas es imprescindible incidir en la formación de bibliotecarios y usuarios sobre el contenido a que dan acceso, sobre su manejo y acerca de sus funcionalidades.

Interesa indicar, para concluir, que para que los servicios de descubrimiento resultasen más efectivos deberían permitir su uso desde otras plataformas aparte de las bibliotecas e incrementar el enlazado a los textos completos de las publicaciones suscritas por la biblioteca y en acceso abierto, una de las fortalezas reconocidas a Google.

En su entrada de 30 de diciembre de 2016, Tay (2016b) se plantea qué sucederá cuando los contenidos estén mayoritariamente disponibles en acceso abierto. ¿Tendrá sentido que cada biblioteca proporcione su servicio de descubrimiento particular? Concluye que en ese escenario el papel de la biblioteca como puerta de entrada para descubrir información declinará pese al valor añadido que puede pro- porcionar personalizando las experiencias de descubrimiento de los miembros de su comunidad académica. Mientras tanto, para seguir en el negocio del descubrimiento de información, los bibliotecarios tienen que ser capaces de dar acceso a los crecientes recursos en acceso abierto de manera eficiente y eficaz.

\section{Notas}

1. Early career researchers: The harbingers of change. Proyecto de tres años de duración (agosto 2015-agosto 2018) subvencionado por Publishing Research Consortium. http://ciber-research.eu/harbingers.html

2. Emerging reputation mechanisms for scholars. European Commission, Joint Research Centre, Institute for Prospective Technological Studies.

http://publications.jrc.ec.europa.eu/repository/bitstream/ JRC94955/jrc94955.pdf

3. Ciber Research Ltd.

http://ciber-research.eu/CIBER_team.html

4. Renaville, François. Discovery tools: A bibliography. https://discoverytoolsbibliography.wordpress.com/byyear/2016-2

5. $b X$ Recommender es un servicio de Ex Libris que ayuda a los usuarios a descubrir bibliografía a partir de un artículo determinado. Se basa en el uso de millones de investigadores y muestra qué otros artículos se usaron junto con el artículo dado.

\section{Bibliografía}

ACRL Research Planning and Review Committee (2012). "2012 top ten trends in academic libraries. A review of the trends and issues affecting academic libraries in higher education". College \& research libraries news, v. 73, n. 6, pp. 311-320.

http://www.oclc.org/research/publications/all/2012-toptrends-academic-libraries.html

Akeroyd, John (2017). "Discovery systems: are they now the library?". Learned publishing, v. 30, n. 1, January, pp. 87-89. https://doi.org/10.1002/leap.1085

Anglada, Lluís (2012). “Bibliotecas universitarias: cabalgando la tecnología, siguiendo al usuario". El profesional de la información, v. 21, n. 6, pp. 553-556.

https://doi.org/10.3145/epi.2012.nov.01

Anglada, Lluís (2014). "Are libraries sustainable in a world of free, networked, digital information?". El profesional de la información, v. 23, n. 6, pp. 603-611.

https://doi.org/10.3145/epi.2014.nov.07

Asher, Andrew D.; Duke, Lynda M.; Wilson, Suzanne (2013). "Paths of discovery: Comparing the search effectiveness of Ebsco Discovery Service, Summon, Google Scholar, and conventional library resources". College \& research libraries, v. 74, n. 5, pp. 464-488.

http://crl.acrl.org/index.php/crl/article/view/16327

Ávila-García, Lorena (2013). Herramientas de descubrimiento en bibliotecas universitarias. Ortiz-Repiso, Virginia y Rodríguez-Mateos, David (dirs.). Trabajo fin de master, Uni- 
versidad Carlos III de Madrid. http://repositorio.ual.es:8080/jspui/bitstream/10835/2569/1/ TFM_Lorena_Avila.pdf

Ávila-García, Lorena; Ortiz-Repiso, Virginia; Rodríguez-Mateos, David (2015). "Herramientas de descubrimiento: ¿una ventanilla única?". Revista española de documentación científica, v. 38, n. 1, e077.

https://doi.org/10.3989/redc.2015.1.1178

Aznar-Lafont, David (2014). "Las herramientas de descubrimiento: los nuevos sistemas de búsqueda global en las bibliotecas académicas". En: Baraibar, Álvaro (ed.). Visibilidad y divulgación de la investigación desde las humanidades digitales. Experiencias y proyectos. Pamplona: Servicio de Publicaciones de la Universidad de Navarra, pp. 253-262. ISBN: 9788480814126.

http://hdl.handle.net/10171/35724

Baldwin, Dee; Kucsak, Michael; Eng, Alice (2012). "Don't touch that string! There went the databases". Information outlook, v. 16, n. 5, pp. 24-32.

http://digitalcommons.unf.edu/library facpub/7

Borrego, Ángel; Anglada, Lluís (2016). "Faculty information behaviour in the electronic environment: Attitudes towards searching, publishing and libraries". New library world, v. 117, n. 3/4, pp.173-185.

https://doi.org/10.1108/NLW-11-2015-0089

Breeding, Marshall (2011). "Discovering Harry pottery barn". Computers in libraries, v. 31, n. 2, pp. 21-23.

https://goo.gl/Ly3Bhm

Breeding, Marshall (2014). "Web-scale discovery services". American libraries, v. 45, n. 1/2, pp. 25-25.

http://americanlibrariesmagazine.org/2014/01/14/webscale-discovery-services

Breeding, Marshall (2015a). The future of library resource discovery: A white paper commissioned by the NISO Discovery to Delivery (D2D) Topic Committee. Baltimore: NISO. ISBN: 9781937522414

http://www.niso.org/apps/group_public/download. php/14487/future_library_resource_discovery.pdf

Breeding, Marshall (2015b). "Informe sobre sistemas bibliotecarios. Hacer operativa la innovación". El profesional de la información, v. 24, n. 4, pp. 485-496.

https://doi.org/10.3145/epi.2015.jul.16

Breitbach, William (2016). "Web-scale discovery: Utopian dream or dystopian nightmare (or maybe something in between)?". En: California academic \& research libraries 2016 conf.

http://conf2016.carl-acrl.org/wp-content/uploads/2016/05/ Breitbach-Web-scale-discovery-FINAL.pdf

Chapman, Suzanne; Dennis, Scott; Folger, Kathleen; Varnum, Ken (2012). "Developing a user-centered article discovery environment". En: Mary-Pagliero Popp; Diane Dallis (eds.). Planning and implementing resource discovery tools in academic libraries. Hershey, PA: IGI Global, pp. 194-224. ISBN: 9781466618213

Chickering, F. William; Yang, Sharon Q. (2014). “Evaluation and comparison of discovery tools: An update". Information technology and libraries, June, pp. 5-30. https://doi.org/10.6017/ital.v33i2.3471

Conrad, Lettie Y. (2017a). "Headlines from the discovery files: Key publications on scholarly content discoverability". Learned publishing, v. 30, n. 1, pp. 31-37. https://doi.org/10.1002/leap.1080

Conrad, Lettie Y. (2017b). "Pathways to a new way of working. Discoverability". Learned publishing, v. 30, n. 1, pp. 3-4. https://doi.org/10.1002/leap.1088

Dempsey, Lorcan (2012). "Thirteen ways of looking at libraries, discovery, and the catalog: scale, workflow, attention". Educause review, December $10^{\text {th }}$.

http://er.educause.edu/articles/2012/12/thirteen-waysof-looking-at-libraries-discovery-and-the-catalog-scaleworkflow-attention

Dempsey, Lorcan (2013). "Discovery vs discoverability..." . En: On libraries, services and networks, January.

http://orweblog.oclc.org/discovery-vs-discoverability

Djenno, Mireille; Insua, Glenda; Gregory, Gwen M.; Brantley, John-Steve (2014). "Discovering usability: Comparing two discovery systems at one academic library". Journal of web librarianship, v. 8, pp. 263-285.

https://works.bepress.com/steve_brantley/23

https://doi.org/10.1080/19322909.2014.933690

Else, Holly (2016). "Libraries becoming invisible to junior scholars". THE, December, 8.

https://www.timeshighereducation.com/news/librariesbecoming-invisible-junior-scholars

Fagan, Jody-Condit; Mandernach, Meris A.; Nelson, Carl S.; Paulo, Jonathan R.; Saunders, Grover (2012). "Usability test results for a discovery tool in an academic library". Information technology and libraries, v. 31, n. 1, March, pp. 83-112. http://ejournals.bc.edu/ojs/index.php/ital/article/ view/1855/1745

https://doi.org/10.6017/ital.v31i1.1855

Faherty, Anna (2016). Academic book discovery, evaluation and access: Insights and opportunities for enhancing the scholarly experience.

https://academicbookfuture.files.wordpress.com/2016/06/ faherty_academic-book-discovery-full-report.pdf

Faiks, Angi; Oberg, Johan; Gabrio, Katy (2012). "WorldCat Local: A transformative solution". En: Mary-Pagliero Popp; Diane Dallis (eds.). Planning and implementing resource discovery tools in academic libraries. Hershey, PA: IGI Global, pp. 608-621. ISBN: 9781466618213

Freund, Leilani; PoehImann, Christian; Seale, Colleen (2012). "From metasearching to discovery: The University of Florida experience". En: Mary-Pagliero Popp; Diane Dallis (eds.). Planning and implementing resource discovery tools in academic libraries. Hershey, PA: IGI Global, pp. 22-43. ISBN: 9781466618213

Gardner, Tracy; Inger, Simon (2016). How readers discover content in scholarly publications. Fernhill: Renew Training. https://goo.gl/icugHc 
Hofmann, Melissa A.; Yang, Sharon Q. (2012). "Discovering what's changed: a revisit of the opacs of 260 academic libraries". Library hi tech, v. 30, n. 2, pp. 253-274.

https://doi.org/10.1108/07378831211239942

Inger, Simon; Gardner, Tracy (2013). "Library technology in content discovery -evidence from a large-scale reader survey". Insights, v. 26, n. 2, pp. 120-127.

http://doi.org/10.1629/2048-7754.75

Kaufmann, Karen; Larsen, Jeanne; De-Salvo, Patricia (2012). "Discovering the discovery tool: The introduction and impact on research and instruction at Seminole State College of Florida". College \& undergraduate libraries, v. 19, n. 2-4, pp. 278-296.

https://doi.org/10.1080/10691316.2012.693432

Kemp, Jan (2012). "Does web-scale discovery make a difference? Changes in collections use after implementing Summon". En: Mary-Pagliero Popp; Diane Dallis (eds.). Planning and implementing resource discovery tools in academic libraries. Hershey, PA: IGI Global, pp. 456-468. ISBN: 9781 466618213

Kennedy, Sean P. (2014). “Uncovering discovery layer services". Public services quarterly, v. 10, n. 1, pp. 54-61. https://doi.org/10.1080/15228959.2014.875788

Lam, Mun-Yee Shirley; Sum, Ming-Ko (2013). “Enhancing access and usage. The OUHK's experience in resource discovery service". En: IFLA World library and information congress, 17-23 August 2013.

Levine-Clark, Michael; McDonald, John; Price, Jason (2014)."The effect of discovery systems on online journal usage: a longitudinal study". Insights, v. 27, n. 3, pp. 249-256. http://doi.org/10.1629/2048-7754.153

Levine-Clark, Michael; Price, Jason; McDonald, John (2013). "Discovery or displacement? A large-scale longitudinal study of the effect of discovery systems on online journal usage". En: Procs of the Charleston library conf. https://doi.org/10.5703/1288284315331

Long Matthew P.; Schonfeld, Roger C. (2014). Ithaka S+R US. Library survey 2013. New York: Ithaka S+R.

http://www.sr.ithaka.org/research-publications/ithaka-srus-library-survey-2013

Mussell, Jessica; Croft, Rosie (2013). "Discovery layers and the distance student: Online search habits of students". Journal of library \& information services in distance learning, v. 7, n. 1-2, pp. 18-39.

National Information Standards Organization (NISO) (2015). ODI Conformance checklists \& statements.

http://www.niso.org/workrooms/odi/conformance

Nicholas, David (2008). "If we do not understand our users, we will certainly fail". En: The e-resources management handbook 1. United Kingdom Serials Group.

http://uksg.metapress.com/content/e2qjfqymrrmg3rqd/ fulltext.pdf

Nicholas, David (dir.) (2016). Early career researchers: the Harbingers of change? Final report from Ciber, Year one (2016).
https://goo.gl/tA89al

Nicholas, David; Boukacem-Zeghmouri, Chérifa; Rodríguez-Bravo, Blanca; Xu, Jie; Watkinson, Anthony; Abrizah, A.; Herman, Eti; Swigon, Marzena (2017). "Where and how early career researchers find scholarly information". Learned publishing, v. 30, n. 1, January, p. 19-29.

https://doi.org/10.1002/leap.108

Nicholas, David; Clark, David; Herman, Eti (2016). “ResearchGate: Reputation uncovered". Learned publishing, v. 29, n. 3, June. https://doi.org/10.1002/leap.1035

Nicholas, David; Herman, Eti; Jamali, Hamid R. (2015). Emerging reputation mechanisms for scholars. European Commission, Joint Research Centre, Institute for Prospective Technological Studies.

http://publications.jrc.ec.europa.eu/repository/bitstream/ JRC94955/jrc94955.pdf

Nicholas, David; Herman, Eti; Jamali, Hamid; RodríguezBravo, Blanca; Boukacem-Zeghmouri, Chérifa; Dobrowolki, Tom; Pouchot, Stephany (2015). "New ways of building, showcasing, and measuring scholarly reputation". Learned publishing, v. 28, n. 3, July, pp. 169-183.

https://doi.org/10.1087/20150303

Niyazov, Yuri; Vogel, Carl; Price, Richard; Lund, Ben; Judd, David; Akil, Adnan; Schwartzman, Josh; Shron, Max (2016) "Open access meets discoverability: Citations to articles posted to Academia.edu". PLoS one, v. 11, n. 2.

https://doi.org/10.1371/journal.pone.0148257

O'Hara, Lisa (2012). “Collection usage pre-and post-Summon implementation at the University of Manitoba libraries". Evidence based library and information practice, v. 7, n. 4, pp. 25-34.

https://doi.org/10.18438/B8DK70

OCLC (2009). Online catalogs: What users and librarians want. Dublin: Ohio: OCLC Online Computer Library Center. https://lccn.loc.gov/2011280465

Ollé, Candela; Borrego, Ángel (2010), “A qualitative study of the impact of electronic journals on scholarly information behavior", Library \& information science research, v. 32, n. 3, pp. 221-228.

https://doi.org/10.1016/j.lisr.2010.02.002

Orduña-Malea, Enrique; Martín-Martín, Alberto, DelgadoLópez-Cozar, Emilio (2016). "ResearchGate como fuente de evaluación científica: desvelando sus aplicaciones bibliométricas". El profesional de la información, v. 25, n. 2, pp. 303310.

https://doi.org/10.3145/epi.2016.mar.18

Perruso, Carol (2016). “Undergraduates' use of Google vs. library resources: A four-year cohort study". College \& research libraries, v. 77, n. 5, pp. 614-630.

https://doi.org/10.5860/crl.77.5.614

Pinto, María; Fernández-Marcial, Viviana; Gómez-Camarero, Carmen (2010), "The impact of information behaviour in academic library service quality: A case study of the science and technology area in Spain". The journal of academic 
librarianship, v. 36, n. 1, pp. 70-78. https://doi.org/10.1016/j.acalib.2009.11.008

Pintos-Ureta, Paula (2016). El etiquetado social y el contenido generado por los usuarios en las bibliotecas universitarias españolas que usan servicios de descubrimiento. Trabajo de fin de grado. Blanca Rodríguez-Bravo (dir.). León: Universidad de León.

Pontis, Sheila; Blandford, Ann; Greifeneder, Eiker; Attalla, Hesham; Neal, David (2015). "Keeping up to date: An academic researcher's information journey". Journal of the Association for Information Science and Technology, v. 68, n. 1, p. 22-35.

https://doi.org/10.1002/asi.23623

Priestner, Andy; Marshall, David (2016). Snapshot: a cultural probe study exploring the research and information behavior of postdocs and PhD students at the University of Cambridge.

https://futurelib.files.wordpress.com/2016/09/the-snapshotproject.pdf

Race, Tammera M. (2012). "Resource discovery tools: Supporting serendipity". En: Mary-Pagliero Popp; Diane Dallis (eds.). Planning and implementing resource discovery tools in academic libraries. Hershey, PA: IGI Global, pp. 139-152. ISBN: 9781466618213

Research Information Network (2009). Creating catalogues: Bibliographic records in a networked world. A RIN report. https://goo.gl/d17zJY

Research Information Network (2011). The value of libraries for research and researchers: A RIN and Rlink report. https://goo.gl/SZAbfk

Rodríguez-Bravo, Blanca; Alvite-Díez, María-Luisa (2011). "An analysis of the use of electronic journals in a Spanish academic context: Developments and profitability". Serials review, v. 37, n. 3, pp. 181-195.

http://www.tandfonline.com/doi/full/10.1080/00987913.2 011.10765381

Rodríguez-Bravo, Blanca; Alvite-Díez, María-Luisa; Olea, Isabel (2015). "La utilización de las revistas electrónicas en la Universidad de León (España): hábitos de consumo y satisfacción de los investigadores". Investigación bibliotecológica, v. 29, n. 66, pp. 17-55.

http://ref.scielo.org/dgpr5s

Rodríguez-Bravo, Blanca; Borges, Maria-Manuel; Fernandes, Alberto-Nuno-Oliveira; Olea-Merino, Isabel; De-Oliveira, Maria-João Carvalho (2013). "Hábitos de consumo y satisfacción con las revistas electrónicas de los investigadores de las universidades de Coimbra, León y Porto". En: Blanca Rodríguez-Bravo, Fernanda Ribeiro, coords. 60 Encontro ibérico Edicic 2013: Globalização, Ciencia e Informação. Porto: Faculdade de Letras da Universidade do Porto: Cetac. Media, pp. 1189-1208.

http://eprints.rclis.org/23189

Rodríguez-Bravo, Blanca; Simões, Maria-da-Graça; DeFreitas, Maria-Cristina-Vieira; Frías, José-Antonio (2015). "La búsqueda de información en herramientas de descubrimiento y opac's: fortalezas y debilidades". En: XII Congreso
ISKO España y II Congreso ISKO España-Portugal. Organización del conocimiento para sistemas de información abiertos. Murcia: Universidad de Murcia.

https://goo.gl/3s7izq

Rodríguez-Bravo, Blanca; Travieso-Rodríguez, Críspulo; Simões, Maria-da-Graça; De-Freitas, Maria-Cristina-Vieira (2014). "Evaluating discovery tools in Portuguese and Spanish academic libraries". En: Wieslaw Babik (ed.) (2014). Knowledge organization in the $21^{\text {st }}$ century: Between historical patterns and future prospects. Procs of the $13^{\text {th }}$ Intl ISKO conf., 19-22 May 2014- Kraków, Poland. Würzburg: ErgonVerlag, pp. 516-523.

http://hdl.handle.net/10174/11782

Rodríguez-Yunta, Luis (2015). "Servicios de descubrimiento basados en un índice centralizado: su expansión en las bibliotecas académicas españolas y futuras líneas de investigación". Anuario ThinkEPI, v. 9, pp. 49-55.

https://doi.org/10.3145/thinkepi.2015.09

Schonfeld, Roger C. (2014). Does discovery still happen in the library? Roles and strategies for a shifting reality. Ithaka $\mathrm{S}+\mathrm{R}$.

https://doi.org/10.18665/sr.24914

Schonfeld, Roger C. (2015). Meeting researchers where they start: Streamlining access to scholarly resources. Ithaka $S+R$. http://www.sr.ithaka.org/wp-content/uploads/2015/03/ SR_Issue_Brief_Meeting_Researchers_Where_They_ Start_032615.pdf

Soules, Aline; Golomb, Liorah; Kelly, James R.; Chen, Barbara (2014). "Navigating the MLA bibliography-redux: Performance across vendor platforms and discovery tools". Journal of library administration, v. 54, n. 2, pp. 107-126. https://doi.org/10.1080/01930826.2014.903367

Spezi, Valérie (2016). "Is information-seeking behavior of doctoral students changing?: A review of the literature (2010-2015)". New review of academic librarianship, v. 22, n. 1, p. $78-106$

https://doi.org/10.1080/13614533.2015.1127831

Spezi, Valérie; Creaser, Claire; Conyers, Angela (2015). "The impact of resource discovery services (RDS) on usage of electronic content in UK academic libraries: Selected results from a UKSG-funded project". Serials review, v. 41, n. 2, pp. 85-99.

https://doi.org/10.1080/00987913.2015.1035991

Tay, Aaron (2016a). "Aggregating institutional repositories: A rethink". Musings about librarianship. https://goo.gl/FX9L4O

Tay, Aaron (2016b). "Library discovery and the open access challenge - Take 2". Mussings about librarianship. https://goo.gl/3S28K1

Tennant, Roy (2009). " $21^{\text {st }}$ century description and access". BiD: textos universitaris de biblioteconomia i documentació, n. 22.

http://bid.ub.edu/22/tennant2.htm

https://doi.org/10.1344/105.000001454

Tenopir, Carol; King, Donald W.; Christian, Lisa; Volentine, Rachel (2015). "Scholarly article seeking, reading, and use: A 
continuing evolution from print to electronic in the sciences and social sciences". Learned publishing, v. 28, n. 2, pp. 93-105. https://doi.org/10.1087/20150203

Thoburn, June; Coates, Annette; Stone, Graham (2012). "Simplifying resource discovery and access in academic libraries: Implementing and evaluating Summon at Huddersfield and Northumbria Universities". En: Mary-Pagliero Popp; Diane Dallis (eds.). Planning and implementing resource discovery tools in academic libraries. Hershey, PA: IGI Global, pp. 580-597. ISBN: 9781466618213

Vaughan, Jason (2012). "Investigations into library webscale discovery services". Information technology and libraries, v. 31, n. 1, pp. 32-82.

https://doi.org/10.6017/ital.v31i1.1916

Warren, John W. (2015). "Zen and the art of metadata maintenance". Journal of electronic publishing, v. 18, n. 3.

https://doi.org/10.3998/3336451.0018.305

Warwick, Clare; Rimmer, Jon; Blandford, Ann; Gow, Jeremy; Buchanan, George (2009). "Cognitive economy and satisficing in information seeking: A longitudinal study of undergraduate information behavior". Journal of the American Society for Information Science and Technology, v. 69, n. 12, pp. 2402-2415.

https://doi.org/10.1002/asi.21179

Way, Doug (2010)."The impact of Web-scale discovery on the use of a library collection". Serials review, v. 36, n. 4, December, pp. 214-220.

http://scholarworks.gvsu.edu/library_sp/9

Wolff, Christine; Rod, Alisa B.; Schonfeld, Roger C. (2016a). Ithaka S+R US faculty survey 2015. New York: Ithaka S+R. https://doi.org/10.18665/sr.277685

Wolff, Christine; Rod, Alisa B.; Schonfeld, Roger C. (2016b). UK survey of academics 2015: Ithaka S+R/Jisc/RLUK. New York: Ithaka S+R.

https://doi.org/10. 18665/sr.282736

Zhu, Julie; Kelley, Jalyn (2015). "Collaborating to reduce content gaps in discovery: What publishers, discovery service providers, and libraries can do to close the gaps". Science \& technology libraries, v. 34, n. 4, p. 315-328. https://doi.org/10.1080/0194262X.2015.1102677

\section{Colección EPI Scholar Libros científicos de Información, Documentación y Comunicación}

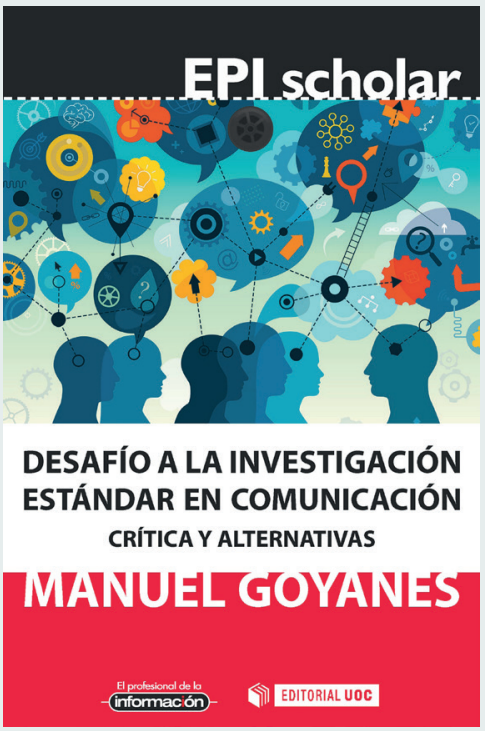

\section{Desafío a la investigación estándar en comunicación. Crítica y alternativas de Manuel Goyanes}

La investigación estándar es la visión legítima y comúnmente aceptada de lo que se considera científico. Es, a grandes rasgos, la buena ciencia. Un tipo de ciencia aplaudida por todos y de la que (casi) todos recogemos sus frutos: permite incrementar la productividad, adquirir estatus $y$, finalmente, notoriedad y acreditaciones. La investigación estándar es algo natural y racional, aunque también el principal factor de deshidratación intelectual de nuestro campo: inunda la investigación con formas y expresiones formularias, silencia la pluralidad de aproximaciones y fomenta el especialismo hasta lo absurdo.

Este libro aborda frontalmente la problemática de la estandarización y sus implicaciones científico-sociales. A través de su lectura, el lector descubrirá cuáles son las normas y valores que regulan la producción de conocimiento, qué hay detrás de la tierra incógnita de nuestras prácticas y disposiciones científicas y cómo desarrollar un tipo de investigación más imaginativa y original. A lo largo de sus páginas muestra cómo es posible pensar e investigar de modo alternativo a través de la problematización de lo dado por sentado, el estilo narrativo y la provocación.

El libro es una llamada de atención para salir de nuestro estado de confort actual. Anima a los investigadores a retar lo establecido y a alcanzar notoriedad e impacto mediante ideas y teorías desafiantes

Goyanes, Manuel (2017). Desafío a la investigación estándar en comunicación. Crítica y alternativas. Barcelona: El profesional de la información, Editorial UOC, colección EPI Scholar n. 7, 164 pp. ISBN: 97884 91166757

Información: Isabel Olea

epi.iolea@gmail.com

http://www.elprofesionaldelainformacion.com/librosEPIScholar.html 